\title{
CONHECIMENTO SOBRE NUTRIÇÃO E CONSUMO DE SUPLEMENTOS EM ACADEMIAS DE GINÁSTICA DE JUIZ DE FORA, BRASIL
}

Original Article

ARTIGO ORIGINAL

Artículo Original

\author{
KNOWLEDGE ON NUTRITION AND SUPPLEMENT CONSUMPTION AT FITNESS CENTERS \\ IN THE CITY OF JUIZ DE FORA, BRAZIL \\ CONOCIMIENTO SOBRE NUTRICIÓN Y CONSUMO DE SUPLEMENTOS EN GIMNASIOS \\ EN LA CIUDAD DE JUIZ DE FORA, BRASIL
}

Fernanda Gargiulo Lopes'
(Nutricionista)
Larissa Loures Mendes'
(Nutricionista)
Mirella Lima Binoti' (Nutricionista)
Natália Pereira de Oliveira'
(Academica em Nutrição)
Nathércia Percegoni' (Nutricionista)

1. Universidade Federal de Juiz de Fora, Departamento de Nutrição, Juiz de Fora, MG, Brasil.

\section{Correspôndencia:}

Universidade Federal de Juiz de Fora. Instituto de Ciências Biológicas/ICB - Departamento de Nutrição, Juiz de Fora, MG, Brasil. Rua: José Lourenço Kelmer, s/n. Juiz de Fora, MG, Brasil. 36036-900. natherciapercegoni@gmail.com

\begin{abstract}
RESUMO
Introdução: O consumo de suplementos alimentares tem ganhado destaque entre desportistas. Contudo, mudanças nos hábitos alimentares são menos expressivas nesta população. Objetivo: Verificar o uso de suplementos alimentares e o conhecimento sobre princípios básicos de nutrição de desportistas frequentadores de academias da cidade de Juiz de Fora, MG, Brasil. Métodos: Estudo transversal, em 19 academias selecionadas por sorteio. A amostra totalizou 348 indivíduos maiores de 18 anos, de ambos os sexos, frequentadores das academias participantes, que concordaram em responder ao questionário contendo questões relacionadas aos dados sociodemográficos, prática de atividade física, conhecimento sobre nutrição e uso de suplementos alimentares. Resultados: Dos participantes, 55\% afirmaram se sentir insatisfeitos com o peso. Ao verificar o conhecimento prévio dos participantes sobre nutrição, $41,5 \%$ afirmaram que os micronutrientes são fornecedores de calorias; $79 \%$ da amostra conhecem a função dos macronutrientes, contudo desconhecem, na totalidade, as principais fontes alimentares dos mesmos. A proteína foi o nutriente que segundo $66 \%$ dos entrevistados deveria estar em maior proporção na dieta. Dos $54 \%$ de participantes que fazem uso de suplementos $43,9 \%$ utilizam um suplemento, $19,1 \%$ dois, 30,5\% de três a quatro e 6,3\% mais de quatro por dia. A orientação para este consumo partiu $34,2 \%$ das vezes do nutricionista, $31 \%$ do educador físico, seguido por outros meios de indicação; o objetivo de ganho de massa magra foi o mais citado (58,2\%). Conclusão: Este trabalho demonstrou elevado consumo de suplementos, insatisfação corporal e equívocos sobre conceitos básicos em nutrição.
\end{abstract}

Palavras-chave: academias de ginástica, suplementos nutricionais, exercício.

\begin{abstract}
Introduction: The consumption of food supplements has been gaining prestige among athletes. However, changes in eating habits are less expressive in this population. Objective: To verify the use of food supplements by athlete-users of fitness centers in the city of Juiz de Fora, MG, Brazil as well as their knowledge of the basic principles of nutrition. Methods: Cross-sectional study in 19 fitness centers selected using a drawing. The sample included 348 participants over age 18 of both sexes who are users of the selected fitness centers and who agreed to answer a questionnaire containing questions related to sociodemographic data, physical activity, knowledge of nutrition, and use of food supplements. Results: Fifty-five percent of participants said they feel dissatisfied with their weight. When checking participants' background knowledge of nutrition, $41.5 \%$ said that micronutrients supply calories, $79 \%$ of the sample knew the function of macronutrients, but all respondents were not aware of the main food sources of these macronutrients. According to $66 \%$ of respondents, protein is the nutrient that should make up the greatest proportion of the diet. Out of the $54 \%$ of participants who use supplements, $43.9 \%$ use one supplement, $19.1 \%$ use two, 30.5\% use three to four and 6.3\% use more than four per day. Of this group, $34.2 \%$ said they were advised on the use of supplements by a nutritionist, while $31 \%$ were advised by a physical educator, followed by other means of receiving recommendations. The most frequently mentioned goal was lean mass gain (58.2\%). Conclusion: This study showed high consumption of supplements, body dissatisfaction and misconceptions about basic concepts of nutrition.
\end{abstract}

Keywords: fitness centers, food supplements, exercise.

\section{RESUMEN}

Introducción: El consumo de suplementos alimenticios ha ganado prominencia entre los deportistas. Sin embargo, los cambios en los hábitos alimenticios son menos expresivos en esta población. Objetivo: Investigar el uso de suplementos alimenticios y el conocimiento sobre los principios básicos de la nutrición de los deportistas de gimnasios en la ciudad de Juiz de Fora, MG, Brasil. Métodos: Estudio transversal en 19 gimnasios seleccionados por sorteo. La muestra consistió de 348 sujetos mayores de 18 años, de ambos sexos, asistentes de los gimnasios participantes, que aceptaran participar y contestar el cuestionario con preguntas relacionadas con 
datos socio-demográficos, la práctica de actividad física, conocimientos de nutrición y el uso de suplementos alimenticios. Resultados: Del total de los participantes, el 55\% dijeron sentirse insatisfechos con el peso. Tras un análisis de los antecedentes de los participantes acerca de la nutrición, el 41,5\% dijeron que los micronutrientes son proveedores de calorías; el 79\% de la muestra tuvieran conocimiento de la función de los macronutrientes, pero ignoran por completo las principales fuentes de los mismos. La proteína fue el nutriente que de acuerdo con el 66\% de los participantes, tiene que estar en mayor proporción en la dieta. Del 54\% de los participantes que usan suplementos, el 43,9\% utilizan un suplemento, el 19,1\% dos; el 30,5\% tres a cuatro y el 6,3\% más de cuatro por día. La orientación de un nutricionista en el consumo de suplementos fue del 34,2\%, el 31\% del educador físico, seguido por otros medios de indicación; el objetivo de aumento de masa magra fue la más citada (58,2\%). Conclusión: Este trabajo demostró un alto consumo de suplementos, de insatisfacción corporal y nociones erróneas acerca de conceptos básicos de nutrición.

Palabras clave: gimnasios, suplementos dietéticos, ejercicio.

\section{INTRODUÇÃO}

Os efeitos positivos do exercício físico bem conduzido, sobre a saúde dos indivíduos são inquestionáveis, sendo um importante fator capaz de influenciar diversos aspectos fisiológicos do organismo e estando positivamente associada a estratégias que levam a mudanças de hábitos alimentares, desencoraja o fumo e a utilização de outras substâncias prejudiciais à saúde, como álcool e drogas ${ }^{1-5}$.

Dentre as diversas modalidades de exercício físico, a musculação ou treinamento resistido tem sido amplamente praticado pelas pessoas que buscam melhor desempenho físico, manutenção ou melhora da qualidade de vida ${ }^{6}$. O número de praticantes de musculação se torna cada vez maior. Adolescentes, adultos e idosos frequentam as academias, seja por iniciativa própria ou por indicação médica, com o objetivo de ganho de massa muscular, perda de peso, fortalecimento muscular, manutenção e promoção à saúde?.

Em 2000, a American Dietetic Association (ADA), a Canadian Dietetic Association (CDA) e o American College of Sports Medicine (ACSM) demonstraram a importante relação entre nutrição e exercício físico, uma vez que a capacidade de rendimento melhora com a nutrição adequada ${ }^{4,8}$. O hábito alimentar adequado é essencial para suprir às demandas energéticas do exercício físico, sendo de suma importância para o desempenho físico 9 .

Sabe-se que as necessidades nutricionais de atletas e praticantes de exercício físico para fins competitivos estão aumentadas. Contudo, a Sociedade Brasileira de Medicina do Exercício e do Esportiva e alguns estudos afirmam que, para os indivíduos que praticam exercícios físicos sem maiores preocupações com o desempenho, uma dieta balanceada de acordo com o recomendado para a população em geral, é suficiente para a manutenção da saúde, possibilitando bom desempenho físico, maior rendimento esportivo, retardo da fadiga e redução da perda de massa muscular ${ }^{10-12}$.

Contudo, o consumo de suplementos alimentares ganhou destaque no meio esportivo não só pelos atletas, mas por desportistas, que buscam no esporte um meio de garantir a saúde e o bem-estar. Porém, esse consumo torna-se indiscriminado, pois, em geral não está relacionado à informação dos usuários sobre a função dos suplementos, bem como seus possíveis efeitos adversos ${ }^{4,13}$.

Cabe ressaltar que o excesso na utilização de suplementos não é o único equívoco cometido por praticantes de exercício físico. Apesar do reconhecimento da população sobre a necessidade de mudanças nos hábitos de vida, demostrada pelo aumento da procura pela prática de exercício físico, as mudanças nos hábitos alimentares são menos expressivas por esta população. A Diretriz da Sociedade Brasileira de Medicina do Exercício e do Esportiva, afirma que esta situação decorre da falta de conhecimento de que uma alimentação balanceada e de qualidade, exceto em situações especiais, atende as necessidades nutricionais de um praticante de exercícios físicos, o que muitas vezes dispensaria o uso de suplementos alimentares ${ }^{10}$.

O presente estudo objetivou verificar o uso de suplementos alimentares por desportistas inseridos em academias, bem como o conhecimento prévio destes a respeito dos princípios básicos de nutrição na cidade de Juiz de Fora, MG, Brasil.

\section{MÉTODOS}

Foi conduzido um estudo transversal, cuja base se constituiu de desportistas frequentadores de academias do município de Juiz de Fora, Minas Gerais, Brasil. Os critérios de elegibilidade adotados foram: idade superior a 18 anos, de ambos os sexos, ser frequentador de uma das academias participantes do estudo, praticar musculação ou outra modalidade de exercício de força ou endurance e concordar em responder ao questionário proposto e assinar o Termo de Consentimento Livre e Esclarecido.

Das 75 academias que constavam na lista telefônica Guiatel e em listas telefônicas online, 25\% foram selecionadas por sorteio ${ }^{4}$, totalizando 19 estabelecimentos para a composição da amostra. Os critérios de inclusão das academias foram: possuir área física variada (ex:: sala de musculação, sala de aulas coletivas, piscina, dentre outros) e oferecerem diversas modalidades, sendo obrigatória a musculação. Portanto, academias que só ofereciam um tipo de atividade (ex.: só dança, pilates, lutas ou só natação) e que ofereciam atividades apenas para uma faixa etária (ex:: só para crianças ou só idosos) e sexo (ex.: só mulheres ou homens) foram excluídas do amostra do estudo.

O tamanho total da amostra $(n=348)$ foi calculado por meio da seguinte equação: $n=\left[\operatorname{EDFF}{ }^{*} N p(1-p)\right] /\left[\left(d^{2} / Z^{2}{ }_{1} a / 2^{*}(N-1)+p^{*}(1-p)\right]\right.$, em que EDFF é o efeito do desenho, sendo utilizado para inquéritos de grupo o valor 1 ; p é a proporção de indivíduos que usam suplementos (36,8\% +/- 5), sendo utilizado como referência a proporção encontrada por Goston; N é o número total da população (10770); d é o limite de confiança (5\%); e Z é o escore padrão da distribuição normal (1,96 para 95\% de confiança), disponível no Website OpenEpi - Versão $3.03^{14}$.

O número de indivíduos por academia foi definido com base no número total de praticantes e na porcentagem que estes representaram na somatória geral de todos os estabelecimentos, multiplicando-se este porcentual pelo total da amostra.

Os alunos foram abordados nos próprios estabelecimentos de maneira aletória e voluntária e responderam um questionário autoaplicável, adaptado dos estudos de Bassit e Malverdi ${ }^{15}$ e Goston ${ }^{4}$. O preenchimento do questionário foi acompanhado para responder possíveis dúvidas dos participantes. 
O questionário abordava questões que envolveram: caracterização sociodemográfica do indivíduo (sexo, idade, escolaridade), hábitos de vida (fumo e consumo de bebidas alcóolicas), prática de exercício físico (tempo e frequência), e questões sobre o conhecimento básico de nutrição (função e fontes dos macronutrientes e micronutrientes). Foi verificado também o uso de suplementos esportivos por parte dos participantes e questões relacionadas à satisfação corporal.

Para caracterizar a amostra, foram apresentadas tabelas de distribuição de frequência. As variáveis quantitativas apresentaram distribuição simétrica e, assim, foram descritas por meio de média e desvio padrão. Para comparar as diferenças entre as frequências foram utilizados Teste Qui-quadrado, Teste Exato de Fisher e para as variáveis com três ou mais categorias utilizou-se o Teste Z $\mathbf{Z}$ de Diferença de Proporções. Os dados foram processados e analisados por meio do programa Statistical Software for Professionals (STATA) versão 13.0 e, para efeito de interpretação, o limite de erro tipo I foi de até $5 \%$.

Para avaliar as questões relacionadas com o conhecimento básico de nutrição no que diz respeito a fontes dos macronutrientes foi desenvolvido um escore baseado no número de acertos para as opções de resposta. Como resultado da pontuação o escore máximo foi de cinco pontos para carboidratos e quatro pontos para proteínas e gorduras indicando acerto de todas as respostas e o escore mínino foi zero pontos indicando nenhum acerto.

Este estudo foi aprovado pelo Comitê de Ética e Pesquisa em Seres Humanos da Universidade Federal de Juiz de Fora, parecer no 820.72. E está em acordo com a resolução 196/96 do Conselho Nacional de Saúde ${ }^{16}$.

\section{RESULTADOS}

Participaram do estudo 348 indivíduos, dos quais 187 (53,7\%) eram homens e 161 (46,3\%) mulheres. Destes, a maioria (68,4\%) são indivíduos jovens com idades entre 18 e 30 anos (tabela 1).

Em relação à escolaridade, metade dos participantes (50,1\%) possuía ensino superior completo ou cursos de pós-graduação lato sensu, mestrado ou doutorado. Além disso, 30,8\% relataram possuir ensino superior incompleto, o que inclui estudantes de graduação. Não se verificou diferença entre homens e mulheres para a escolaridade (tabela 1).

No que diz respeito a pratica de atividade física, mais da metade dos participantes, 61,3\% praticam atividade física há mais de um ano e $73,2 \%$ dos indivíduos relataram uma frequência de prática de atividade física semanal de três a cinco vezes (tabela 2). Homens e mulheres apresentaram padrão diferente $(p<0,05)$ em relação à frequência em que praticam atividade física durante a semana. Mais mulheres realizam atividade física menos de três vezes por semana e mais homens fazem atividade mais de cinco vezes por semana (tabela 2 ).

Do total de participantes, mais da metade (55\%) afirmou se sentir

Tabela 1. Análise descritiva das variáveis idade e escolaridade em relação ao sexo. Juiz de Fora, Minas Gerais, 2014 ( $n=348)$.

\begin{tabular}{c|c|c|c|c|c|c}
\hline \multirow{2}{*}{ Variáveis } & \multicolumn{4}{|c|}{ Sexo } & \multicolumn{2}{c}{ Total } \\
\cline { 2 - 5 } & \multicolumn{2}{|c|}{ Masculino } & \multicolumn{2}{c|}{ Feminino } & \multicolumn{2}{c}{} \\
\cline { 2 - 6 } & $\mathbf{n}$ & $\%$ & $\mathbf{n}$ & $\%$ & $\mathbf{n}$ & $\%$ \\
\hline Idade (anos) & & & & & & \\
\hline $18-30$ & 131 & 70,05 & 107 & 66,46 & 238 & 68,39 \\
\hline $31-49$ & 43 & 22,99 & 42 & 26,09 & 85 & 24,43 \\
\hline$\geq 50$ & 13 & 6,95 & 12 & 7,45 & 25 & 7,18 \\
\hline Escolaridade & & & & & & \\
\hline $1^{\circ}$ Grau & 1 & 0,53 & 3 & 1,88 & 4 & 1,15 \\
\hline $2^{\circ}$ Grau & 38 & 20,32 & 24 & 15,00 & 62 & 17,87 \\
\hline Superior incompleto & 63 & 33,69 & 44 & 27,50 & 107 & 30,84 \\
\hline Superior completo & 46 & 24,60 & 50 & 31,25 & 96 & 27,67 \\
\hline Pós-graduado & 39 & 20,86 & 39 & 24,38 & 78 & 22,48 \\
\hline
\end{tabular}

insatisfeito em relação ao peso, sendo que as mulheres $(61,2 \%)$ apresentaram maior percentual de insatisfação $(p<0,05)$ com o peso que os homens (49,7\%) (tabela 3).

Ao verificar a percepção da imagem corporal em relação ao peso real, a maioria (48,2\%) acreditava estar com o peso acima do ideal, enquanto $20,1 \%$ se auto-classificou com o peso abaixo do ideal. Neste sentido, verificou-se diferença significativa entre homens e mulheres $(p<0,05)$, sendo que no grupo dos homens, uma grande parcela se classificou como abaixo do ideal (tabela 3).

Em relação ao consumo de bebidas alcoólicas, 65,6\% relatavam consumir, sendo a proporção de consumo entre homens (68\%) e muIheres $(61,5 \%)$ semelhante. Ao verificar a frequência de consumo, mais da metade $(54,1 \%)$ dos participantes fazia uso de bebidas alcóolicas de uma a duas vezes por semana, sendo que não houve relato de consumo de cinco a mais vezes por semana. Quanto ao tabagismo; $86,2 \%$ relataram não fumar, não havendo diferença significativa entre homens e mulheres.

Ao verificar o conhecimento prévio dos participantes no que diz respeito à nutrição foi possível perceber que 79\% da amostra conhecia a função dos macronutrientes, não havendo diferença nestes conhecimentos por sexo.

Porém, quando questionados sobre fornecimento de energia pelos micronutrientes, 41,5\% afirmaram que os micronutrientes são fornecedores de energia/calorias, indicando elevado percentual erro.

Com relação ao conhecimento sobre as fontes alimentares dos macronutrientes carboidrato, proteína e lipídio, foram encontrados resultados similares.

No que diz respeito aos carboidratos, observou-se menor escore de acerto, sendo que $46,5 \%$ indivíduos assinalaram como fontes deste macronutriente três das cinco fontes apresentadas no inquérito (figura 1). Quando se compara este conhecimento entre homens e mulheres, verifica-se que há diferença $(p<0,05)$ para a categoria de três acertos, sendo a proporção maior para mulheres (56,5\%) em relação aos homens (37\%).

Tabela 2. Análise descritiva do padrão de prática de atividade física em relação ao sexo. Juiz de Fora, Minas Gerais, 2014 ( $n=348)$.

\begin{tabular}{c|c|c|c|c|c|c}
\hline \multirow{2}{*}{ Variáveis } & \multicolumn{4}{|c|}{ Sexo } & \multicolumn{2}{c}{ Total } \\
\cline { 2 - 6 } & Masculino & \multicolumn{2}{c}{ Feminino } & \multicolumn{2}{c}{} \\
\cline { 2 - 7 } & $\mathbf{n}$ & $\%$ & $\mathbf{n}$ & $\%$ & $\mathbf{n}$ & $\%$ \\
\hline Tempo de prática & & & & & & \\
\hline$<1$ mês & 15 & 8,02 & 22 & 13,75 & 37 & 10,66 \\
\hline 1 a 6 meses & 22 & 11,76 & 39 & 24,38 & 61 & 17,58 \\
\hline 7 meses a 1 ano & 19 & 10,16 & 17 & 10,62 & 36 & 10,37 \\
\hline$>1$ ano & 131 & 70,05 & 82 & 51,25 & 213 & 61,38 \\
\hline Frequência semanal & & & & & & \\
\hline$<3 x /$ sem & 7 & 3,74 & 18 & 11,25 & 25 & 7,2 \\
\hline 3 a 5x/sem & 133 & 71,12 & 121 & 75,62 & 254 & 73,2 \\
\hline$>5 x /$ sem & 47 & 25,13 & 21 & 13,12 & 68 & 19,6 \\
\hline
\end{tabular}

Tabela 3. Análise descritiva da autoclassificação do peso corporal em relação ao sexo. Juiz de Fora, Minas Gerais, 2014 ( $n=348)$.

\begin{tabular}{c|c|c|c|c|c|c}
\hline \multirow{2}{*}{ Variáveis } & \multicolumn{4}{|c|}{ Sexo } & \multicolumn{2}{c}{ Total } \\
\cline { 2 - 6 } & Masculino & \multicolumn{2}{c}{ Feminino } & \multicolumn{2}{c}{} \\
\cline { 2 - 7 } & $\mathbf{n}$ & $\%$ & $\mathbf{n}$ & $\%$ & $\mathbf{n}$ & $\%$ \\
\hline Satisfação com o peso & & & & & & \\
\hline Sim & 94 & 50,27 & 62 & 38,75 & 156 & 44,96 \\
\hline Não & 93 & 49,73 & 98 & 61,25 & 192 & 55,05 \\
\hline Percepção do peso & & & & & & \\
\hline Acima do Ideal & 74 & 39,57 & 94 & 58,39 & 168 & 48,28 \\
\hline Ideal & 63 & 33,69 & 47 & 29,19 & 110 & 31,61 \\
\hline Abaixo do Ideal & 50 & 26,74 & 20 & 12,72 & 70 & 20,11 \\
\hline
\end{tabular}


Nas questões envolvendo fontes de proteínas, 41,3\% dos indivíduos assinalaram três das fontes alimentares apresentadas, deixando de lado apenas uma e 35,3\% acertaram todas as quatro fontes de proteínas, o que demonstra margem de acerto representativa (figura 1).

Para as fontes alimentares de lipídios/gorduras, também foi encontrado elevado percentual de acerto nas respostas. Mais da metade da amostra 52\% acertou todas as fontes alimentares e 33,6\% dos indivíduos assinalou três das quatro fontes sugeridas no questionário (figura 1). Não houve diferença de conhecimento quanto às fontes de proteína e gordura entre homens e mulheres.

Quando questionados sobre a composição da dieta, com relação à distribuição de macronutrientes, 66\% dos participantes assinalaram a proteína como sendo o nutriente que deve ser mais consumido na dieta, seguido dos carboidratos (33\%) e gorduras (1\%) (figura 2). Neste caso, os homens apresentaram percentual de acerto maior que as mulheres, $38,4 \%$ e $26,7 \%(p<0,05)$, respectivamente.

Com relação ao consumo de suplementos, $54 \%$ dos participantes relataram fazer o uso de pelo menos um suplemento alimentar diariamente (figura 3), sendo que os homens apresentaram frequência de consumo maior que as mulheres, 57,8\% e 31,2\% ( $p<0,05)$, respectivamente.

Quanto ao número de suplementos consumidos, foi possível observar que $43 \%$ dos indivíduos que faziam o uso de suplementos utilizava somente um suplemento por dia, sendo que a maioria (64\%) das mulheres se enquadrou nessa categoria. Dos homens, 19,6\% utilizam dois suplementos; $36,4 \%$ utilizam de três a quatro suplementos e 9,3\% utilizam mais de quatro suplementos diariamente, demonstrando maior consumo de suplementos pelos homens em relação às mulheres $(p<0,05)$. Dentre as mulheres, nenhuma relatou consumir mais de quatro suplementos por dia (tabela 4).

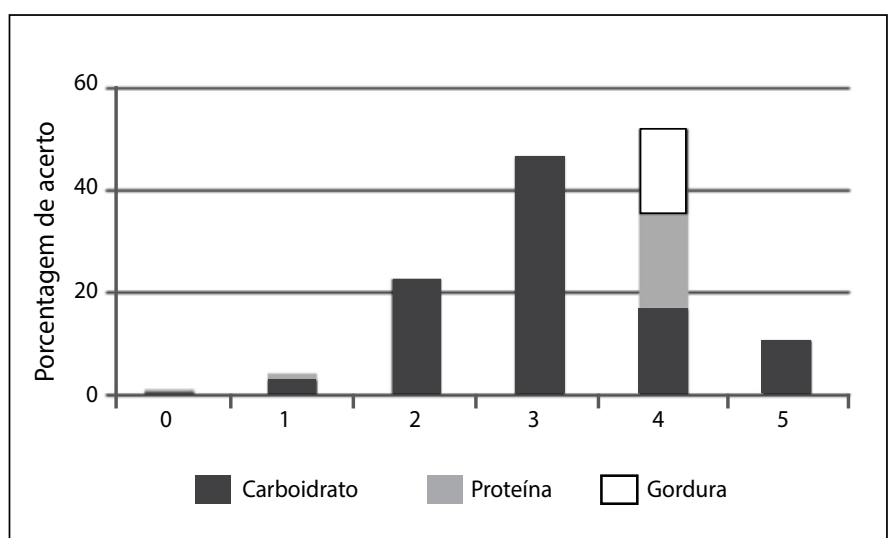

Figura 1. Percentual de escore de acerto das fontes alimentares de macronutrientes. Juiz de Fora, Minas Gerais, 2014 ( $n=348)$.

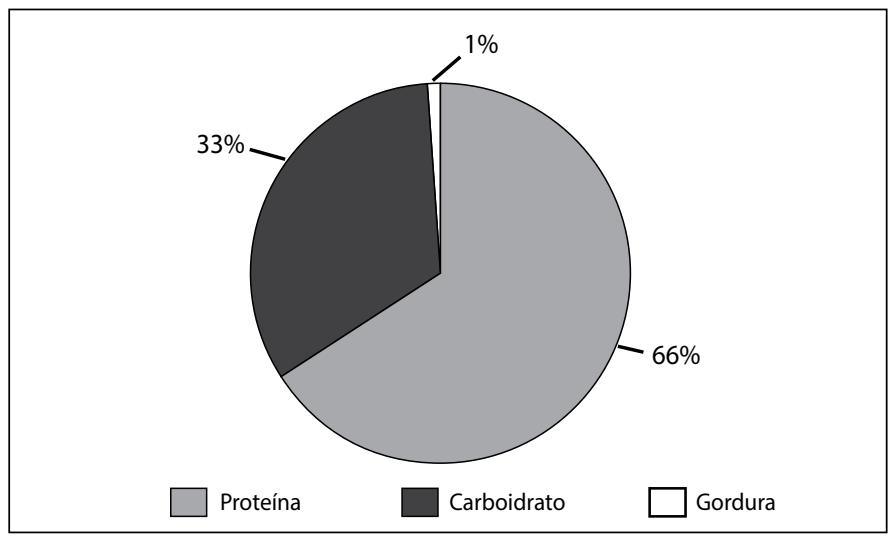

Figura 2. Conhecimento sobre a proporção adequada de macronutrientes na dieta. Juiz de Fora, Minas Gerais, 2014 ( $n=348)$.

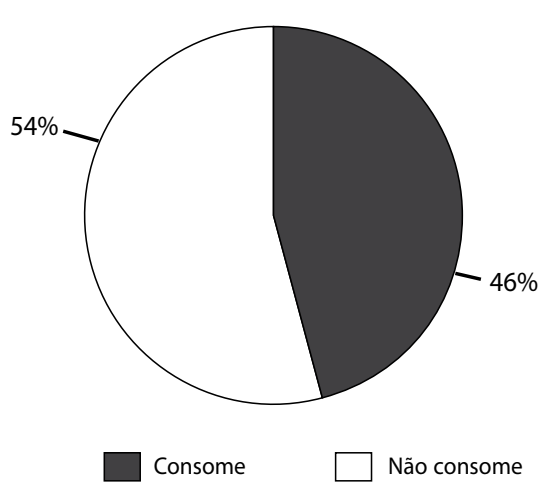

Figura 3. Consumo de suplementos alimentares. Juiz de Fora, Minas Gerais, $2014(n=348)$.

Tabela 4. Consumo de suplementos - Juiz de Fora, Minas Gerais, 2014 ( $n=348)$.

\begin{tabular}{c|c|c|c|c|c|c}
\hline \multirow{2}{*}{ Variáveis } & \multicolumn{4}{|c|}{ Sexo } & \multicolumn{2}{c}{ Total } \\
\cline { 2 - 6 } & \multicolumn{2}{|c|}{ Masculino } & \multicolumn{2}{c}{ Feminino } & \multicolumn{2}{c}{} \\
\cline { 2 - 6 } & $\mathbf{n}$ & $\%$ & $\mathbf{n}$ & $\%$ & $\mathbf{n}$ & $\%$ \\
\hline No de suplementos utilizados & & & & & & \\
\hline 1 suplemento & 37 & 34,58 & 32 & 64,00 & 69 & 43,95 \\
\hline 2 suplementos & 21 & 19,63 & 9 & 18,00 & 30 & 19,11 \\
\hline 3 a 4 suplementos & 39 & 36,45 & 9 & 18,00 & 48 & 30,57 \\
\hline$>$ 4 suplementos & 10 & 9,35 & 0 & 0 & 10 & 6,37 \\
\hline
\end{tabular}

Quando questionados sobre a indicação para o uso dos suplementos esportivos, observou-se que 34,2\% de indicações partiram do profissional nutricionista, apesar de grande parte da amostra (59,7\%) nunca ter recebido orientação nutricional até o momento da entrevista; $31 \%$ das indicações foram realizadas por profissionais de educação física, seguidos das pessoas que consomem suplementos sem orientação $15,2 \%$, influenciados pelo comerciante da loja de suplementos $8,8 \%$, $2,5 \%$ indicados por médicos e $8,2 \%$ por outros. Cabe ressaltar ainda que mais da metade dos participantes (58,2\%) consome suplementos com o objetivo de ganho de massa magra, 5,7\% tem como objetivo apenas o emagrecimento e 26,6\% apresentam mais de dois objetivos concomitantemente, como o ganho de massa magra e o emagrecimento.

\section{DISCUSSÃO}

O perfil de frequentadores das academias de ginástica avaliado compõe-se de indivíduos na faixa etária de 18 a 35 anos de idade, com alto grau de escolaridade e sem diferença significativa entre os sexos, o que se aproxima do perfil de praticantes de atividade física de outros estudos ${ }^{17-20}$

Grande parte da amostra (61,3\%) praticava atividade física há mais de um ano. Estudo realizado por Soares et al., revelou haver alta prevalênica de indíviduos que frequentam a academia em um período maior que 12 meses $^{21}$. Foi possível observar ainda, que homens frequentam academia mais vezes por semana quando comparado às mulheres.

No estudo de Lima e Bezerra22 o tabagismo foi referido por apenas 9,8\% da população estudada, o que se aproxima dos resultados encontrados no presente estudo onde verificou-se que $86,2 \%$ não fumam e nunca fumaram. Contudo, em relação ao consumo de álcool, a população estudada apresentou um percentual de consumo mais elevado $(65,6 \%)$ do que o relatado no estudo supracitado que foi de $53,7 \%$.

Os conhecimentos sobre nutrição relatados pelos participantes do presente estudo indicam que esses indivíduos apresentam bom conhecimento em relação a alguns conceitos de nutrição, com elevado percentual de acerto no que diz respeito às funções dos macronutrientes e à identificação das fontes alimentares dos mesmos. Contudo, 
outros conceitos como o não fornecimento de caloria por parte dos micronutrientes e o nutriente que deve estar em maior proporção na dieta apresentaram elevado percentual de erro.

Ao avaliar o conhecimento nutricional dos praticantes de musculação da Secretaria e Esporte do Distrito Federal, Paes ${ }^{7}$ verificou que os indivíduos possuiam conhecimento no que se refere à identificação dos alimentos fontes de macronutrientes e pouco conheciam sobre os micronutrientes, com 49\% deles acreditando que os micronutrientes fornecem energia para o organismo, o que se assemelha aos resultados encontrados na presente pesquisa.

Quando questionados a respeito do macronutriente que deve estar em maior proporção na dieta, 66\% dos participantes do presente estudo assinalaram a proteína e $33 \%$ assinalaram o carboidrato. Este dado talvez se justifique pela valorização que os suplementos à base de proteína têm no ambiente de academia. Diversos estudos mostram que os suplementos mais utilizados são os proteicos ${ }^{4,23-26}$.

Este é um erro cometido não só por praticantes de atividade física como também por atletas profissionais. Foi o que verificou Alaunyte et al. ${ }^{27}$, que ao avaliar os conhecimentos sobre nutrição por atletas de Rugby da Super Liga Inglesa, constataram que mesmo apresentando um bom conhecimento, os atletas consumiam fontes de carboidratos ocasionalmente, demonstrando um certo receio quanto ao consumo deste macronutriente.

No Brasil os estudos apontam perfis diferentes no consumo de suplementos, contudo, esse consumo ainda se caracteriza como elevado em grande parte dos levantamentos feitos. No presente estudo a frequência de consumo foi de 54\%. Em Recife, Pereira e Cabral ${ }^{13}$ encontraram uma prevalência de consumo de 38,3\%, dado bastante parecido com o de Belo Horizonte, com 36,8\% de prevalência no consumo de suplementos ${ }^{4}$. Já em Sete Lagoas, MG, Brasil, a prevalência de consumo foi de $62 \%{ }^{26}$ e em Ribeirão Preto, SP, Brasil, o consumo foi de $53 \%{ }^{27}$, se assemelhando ao resultado encontrado.

Estimativas mundiais do uso de suplementos alimentares por atletas ficam entre 40 e $80 \%$ da população. Um estudo com dançarinos profissionais de 53 países apontou um uso de suplemento por $48 \%$ dos atletas, dado que também se aproxima do encontrado no presente estudo 23,28 .

Goston ${ }^{4}$ comparou o consumo entre homens e mulheres e assim como os resultados encontrados no presente estudo, o consumo de suplementos por homens foi maior que o consumo pelas mulheres. Johann e Berleze ${ }^{25}$ verificaram que os consumidores de suplementos alimentares buscavam mais o ganho de peso em relação às pessoas que não consumiam o que talvez aponte o motivo do consumo mais expressivo de suplementos por parte dos homens, uma vez que a maioria destes se auto-classifica com o peso abaixo do ideal.

Com relação ao número de suplementos consumidos, Hallak et al. ${ }^{19}$, contataram que $44,2 \%$ dos entrevistados que faziam o uso de suplementos consumiam apenas um tipo, o que se aproxima dos achados no presente estudo, no qual foi verificada a frequência de 43,95\%. Contudo, o consumo de mais de um suplemento divergiu dos achados pelos autores citatos. Sendo que, o consumo de dois suplementos foi menor nos nossos achados (19,1\% contra $27,1 \%)$, bem como o consumo concomitante de mais de quatro suplementos (6,3 contra $12,4 \%)$. Cabe ressaltar que o consumo de três a quatro suplementos foi maior com 30,5\% contra $13,1 \%$.

Fayh et al. ${ }^{23}$, assim como em nossos achados, constataram um consumo concomitante de suplementos mais frequente entre homens, sendo que dos 33 indivíduos que relataram ingerir mais do que um suplemento, apenas três eram mulheres.

Fontes e Navarro ${ }^{26}$, apontaram que a indicação para o uso de suple- mento alimentar, partindo do nutricionista foi reduzida em relação aos outros profissionais, com $17 \%$ e $12 \%$ respectivamente, o que diverge dos achados do presente estudo. Contudo, é preciso destacar que, apesar de termos o nutricionista como o profissional mais citado na indicação para o uso do suplemento, com 34,1\%, o percentual de indicação por profissionais de outras áreas e o consumo sem orientação também foi elevado, não sendo esta a prática ideal, uma vez que o consumo de suplementos deve vir associado a uma alimentação equilibrada, e o nutricionista é o profissional capacitado para prescrição da dieta e promoção da alimentação saudável.

Apesar do presente estudo não apresentar diferença do conhecimento sobre nutrição entre os sexos, estudos com atletas profissionais demonstraram um melhor perfil e conhecimento alimentar por mulheres ${ }^{29,30}$. Segundo Jacobson et al. ${ }^{30}$, isto se deu pelo fato de que para a maioria das mulheres a orientação nutricional partiu prioritariamente de um nutricionista.

No esporte profissional, uma maior participação do nutricionista esportivo na formação e orientação dos hábitos alimentares foi constatado por Devlin e Belski ${ }^{31}$, que em suas análises observaram que 98\% dos atletas de futebol Australiano tinham a orientação nutricional de um nutricionista.

Hirschbruch et al. ${ }^{32}$, apontaram que indivíduos usuários de suplementos se exercitam para ganhar massa muscular e perder gordura havendo associação entre esses objetivos e o consumo de suplementos o que corrobora com os dados encontrados no presente trabalho, com $58,2 \%$ da população apresentando como objetivo o ganho de massa magra. Contudo, Alvarez et al. ${ }^{33}$, constataram que 56\% dos entrevistados apresentavam como objetivo a perda de peso, o que diverge bastante dos dados encontrados, uma vez que apenas 5,7\% da população apresentou como objetivo único a perda de peso.

Pelo descrito acima, verifica-se que este estudo se aproxima dos demais achados em outras pesquisas, apresentando um elevado consumo de suplementos e insatisfação com a imagem corporal por essa população, e alguns equívocos no que diz respeito aos conhecimentos básicos sobre nutrição. Foi possível ainda constatar um elevado consumo de bebidas alcóolicas, mesmo que em menor frequência, por uma população que, em tese, se preocupa com a estética corporal.

Tais dados demonstram que, apesar da procura pela atividade física vir associada às mudanças de hábitos alimentares e à busca por um estilo de vida mais saudável; o uso de suplementos alimentares de forma indiscriminada e sem indicação do profissional de nutrição, ainda é uma realidade. Além deste fato, a ausência de conhecimento sobre fontes alimentares dos principais nutrientes para a manutenção da saúde do indivíduo, apresentou-se como um achado impactante nas práticas alimentares e de consumo de suplementos observada.

Por fim cabe ressaltar algumas limitações e potencialidades do presente estudo. No que diz respeito às limitações ressalta-se que a listagem oficial das academias da cidade Juiz de Fora não foi disponibilizada pelos órgãos responsáveis. A temporalidade que é inerente ao desenho transversal, e o uso de questionário com perguntas relativas ao estilo de vida que dependem da memória e do interesse dos indivíduos em relação aos temas de investigação torna o estudo passível de vieses, que podem limitar os resultados encontrados.

Em relação às potencialidades é importante ressaltar que são escassos os estudos no Brasil sobre o conhecimento prévio de princípios básicos da nutrição por praticantes de exercício físico. Tal fato é de suma relevância, pois o desconhecimento sobre alimentação pode induzir ao consumo de suplementos alimentares de forma indiscriminada. Desta forma, mais estudos que visem avaliar o tema devem ser conduzidos. 


\section{CONCLUSÃO}

Há elevado consumo de suplementos alimentares, insatisfação corporal e equívocos sobre conceitos básicos em nutrição entre frequentadores de academias de Juiz de Fora. É necessária a conscientização dos praticantes de exercício físico, sobre alimentação equilibrada e hábitos de vida saudáveis para a manutenção da saúde e da estética corporal desejada, independentemente do uso de suplementos alimentares.

Todos os autores declararam não haver qualquer potencial conflito de interesses referente a este artigo.

\section{REFERÊNCIAS}

1. Nicastro H, Dattilo M, Santos TR, Padilha HVG, Zimberg IZ, Crispim CA, et al. Aplicação da escala de conhecimento nutricional em atletas profissionais e amadores de atletismo. Rev Bras Med Esporte. 2008;14(3):205-8.

2. Mello MT, Boscolo RA, Esteves AM, Tufik, S. O exercício físico e os aspectos psicobiológicos. Rev Bras Med Esporte. 2005;11(3):203-7.

3. Crozeta C, Oliveira GK. Análise do perfil alimentar de mulheres com sobrepeso, praticantes de treinamento de força em academias de Curitiba-PR. Rev Bras Nutr Esportiva. 2009;3(17):432-41.

4. Goston JL. Prevalência do uso de suplementos nutricionais entre praticantes de atividade física em academias de Belo Horizonte: fatores associados [dissertação]. Belo Horizonte: Universidade Federal de Minas Gerais, Faculdade de Farmácia; 2008.

5. Dumith SC. Physical activity in Brazil: a systematic review. Cad Saúde Pública. 2009;3(25):S415-S26

6. Machado MC, Paixão MPCP. Estudo de caso com praticantes de musculação. Rev Bras Nutr Esportiva. 2012;6(36):477-85.

7. Paes SR. Conhecimento nutricional dos praticantes de musculação da secretaria de estado de esporte do Distrito Federal. Rev Bras Nutr Esportiva. 2012;6(32):105-11.

8. Zilch MC, Soares BM, Bennemann GD, Sanches FLF, Cavazzotto TG, Santos EF. Análise da ingestão de proteínas e suplementação por praticantes de musculação nas academias centrais da cidade de Guarapuava - PR. Rev Bras Nutr Esportiva. 2012;6(35):381-8.

9. Pacheco BM, Filho ADR, Santini E. Impacto da prática regular de exercício físico sobre aspecto alimentar. Rev Bras Nutri Esportiva. 2012;6(35):376-80

10. Sociedade Brasileira de Medicina do Esporte e do Exercício. Modificaçōes dietéticas, reposição hídrica, suplementos alimentares e drogas: comprovação de ação ergogênica e potenciais riscos para a Saúde. Rev Bras Med Esporte. 2009;15(3):3-12.

11. Carvalho T, Mara LS. Hidratação e nutrição no esporte. Rev Bras Med Esportiva. 2010;16(2):144-8.

12. Costa WSA. Avaliação do estado nutricional e hábitos alimentares de alunos praticantes de atividade física de uma academia do município de São Bento do Uno - PE. Rev Bras Nutr Esportiva. 2012;6(36):464-9

13. Pereira JMO, Cabral P. Avaliação dos conhecimentos básicos sobre nutrição de praticantes de musculação em uma academia da cidade de Recife. Rev Bras Nutr Esportiva. 2007;1(1):40-7.

14. Kelvin MS, Andrew D, Minn MS. OpenEpi: A web-based epidemiologic and statistical calculator for public health. Pub Health Reports. 2009;124(3):471-4

15. Bassit RA, Malverdi MA. Avaliação nutricional de triatletas. Rev Paul Educ Fís. 1998;12(1):42-53.

16. Ministério da Saúde, Conselho Nacional de Saúde. Resolução n. 196 de 10 de outubro de 1996. Aprova as diretrizes e normas regulamentadoras de pesquisas envolvendo seres humanos. Informe epidemiológico do SUS. Brasil: Ministério da Saúde; 1996

17. Silva AA, Fonseca NSL, Gagliardo IC. A associação da orientação nutricional ao exercício de força na hipertrofia muscular. Rev Bras de Nutr Esportiva. 2012;6(3):389-97.
18. Duran ACFL, Latorre MRD, Florindo AA, Jaime PC. Correlação entre consumo alimentar e nível de atividade física habitual de praticantes de exercícios físicos em academia. Rev Bras Ciênc Mov. 2004;12(3):15-9

19. Hallak A, Fabrini S, Peluzio MCG. Avaliação do consumo de suplementos nutricionais em academias da zona sul de Belo Horizonte, MG, Brasil. Rev Bras Nutr Esportiva. 2007;1 (2):55-60

20. Adam BO, Fanelli C, Souza ES, Stulbach TE, Monomi PY. Conhecimento nutricional de praticantes de musculação de uma academia da cidade de São Paulo. Rev Bras Nutr Esportiva. 2013;2(2):24-36.

21. Soares LP, Pita JSL, Mgalhães SS. Perfil dietético, estado nutricional e nível de atividade física em praticantes de exercícios físicos das academias de Vitória da Conquista - BA. Rev Bras Nutr Esportiva. 2012;6(35):343-52.

22. Lima RS, Bezerra TAL. Avaliação do consumo alimentar e utilização de suplementos nutricionais por praticantes de musculação em Caruaru - PE [monografia]. Curuaru, PE: Faculdade do Vale do Ipojuca; 2008

23. Fayh APT, Silva CV, Jesus FRD, Costa GK. Consumo de suplementos nutricionais por frequentadores de academias da cidade de Porto Alegre. Rev Bras Ciênc Esporte. 2013:35(1):27-37.

24. Gomes SG, Degiovanni GC, Garlipp MR, Chirello PG, Jordão Jr AA. Caracterização do consumo de suplementos nutricionais em praticantes de atividade física em academias. Medicina (Ribeirão Preto) 2008;41(3):327-31.

25. Johann J, Berleze KJ. Estado nutricional e perfil antropométrico de frequentadores de academias de ginástica, usuários ou não de suplementos de cinco municípios do interior do Rio Grande do Sul. Rev Bras Nutr Esportiva. 2010:4(21):197-208.

26. Fontes AMS, Navarro F. Consumo de suplementos nutricionais por praticantes de atividades fisícas em academias de Sete Lagoas - MG. Rev Bras Nutr Esportiva. 2010;4(24):515-23.

27. Alaunyte I, Perry $J$, Aubrey T. Nutritional knowledge and eating habits of professional rugby league players: does knowledge translate into practice? J Int Soc Sports Nutr. 2015;12(18):1-7.

28. Brown D, Wyon M. An international study on dietary supplementation use in dancers. Med Probl Perform Art. $2014: 29(4): 229-34$

29. Spronk L, Heaney SE, Prvan T, O'Connor HT. Relationship between general nutrition knowledge and dietary quality in elite athletes. Int J Sport Nutr Exerc Metab. 2015;25(3):243-51.

30. Jacobson BH, Sobonya C, Ransone J. Nutrition practices and knowledge of college varsity athletes: a follow-up. J Strength Cond Res. 2001;15(1):63-8.

31. Devlin BL1, Belski R. Exploring General and Sports Nutrition and food Knowledge in Elite Male Australian Athletes. Int J Sport Nutr Exerc Metab. 2015;25(3):225-32.

32. Hirschbruch MD, Fisberg M, Mochizuk L. Consumo de suplementos por Jovens Frequentadores de Academias de Ginástica em São Paulo. Rev Bras Med Esporte. 2008;14(6):539-43.

33. Alvares FGG, Reis JCF, Ennes MG. Avaliação prática de atividade aeróbia e nível de conhecimento de seus praticantes. Rev de Educ Fís. 2008;140(1):13-9. 